\title{
Caracterizaciòn de la fonética y escritura de la letra " $A$ " en señales cerebrales usando transformada wavelet
}

\author{
German Arley Portilla González 1:, Luis Enrique Mendoza 2. \\ .,Saury Thomas $\mathbf{M}^{3}$ \\ 1,2Grupo de Ingeniería Biomédica, GIBUP.Universidad de Pamplona. \\ ${ }^{3}$ Grupo de Investigaciòn INGAPO..Universidad de Pamplona.Pamplona, Norte de \\ Santander, Colombia
}

Resumen: La afasia motriz afecta únicamente el lenguaje, y el área cognitiva queda intacta, esta falla cerebral se presenta sobre el área de broca, donde se manejan los impulsos aferentes de la articulación y modulación del lenguaje. Este trabajo muestra la obtención usando wavelet de patrones característicos de señales encefalografías provenientes de: fonética y escritura de la letra $A$, esto permitirá en un futuro tener una alternativa de comunicación distinta a pronunciar palabras. Este trabajo se presenta debido a las gran cantidad accidentes cerebrovasculares que afectan la capacidad de dialogo. Mediante la toma de señales electroencefalografías (SEEG) en puntos específicos se puede lograr caracterizar procesos de: gesticulación e interpretación de letras. El estudio se aplicó sobre 10 sujetos con edades de 24 y 25 años, la toma fue en condiciones similares en estado de vigilia. Se registraron 20 señales de la letra pronunciada, 20 de la letra escrita y 5 del sujeto en reposo. Se demostró que existen patrones diferentes que caracterizan la pronunciación y escritura de la letra "A" en una representación de SEEG.

Palabras clave: Afasia, fonética, encefalografícas, procesamiento.

\section{A characterization of phonetics and writing of the letter "A" in cerebral signals using transformed wavelet}

\begin{abstract}
The motive aphasia affects only the language, and the cognitive area remains intact, this cerebral fault appears on the area of reel, where they handle the impulses afferents of the joint and modulation of the
\end{abstract}


113

language. This work shows the obtaining using wavelet of bosses typical of signs encephalographyc's from of: phonetics and writing of the letter To, this will allow in a future to have an alternative of different communication to words declare. This work appears due to great quantity cerebro-vascular accidents that affect the capacity of dialog. By means of the capture of signs encephalographyc's (SEEG) in specific points it is possible to manage characterize processes of: gesticulation and interpretation of letters. The study was applied on 10 subjects by ages of 24 and 25 years, the capture was in similar conditions in state of wake. There were registered 20 signs of the pronounced letter, 20 of the written letter and 5 of the subject in rest. There was demonstrated that you exist bosses who characterize the pronunciation and writing of the letter "A" in SEEG's representation.

Keywords: Aphasias, Phonetics, Encephalography's, Processing.

*Para citar este artículo: Portilla González GA., Mendoza LE ., Thomas M S. Caracterizaciòn de la fonética y escritura de la letra " $A$ " en señales cerebrale usando transformada wavelet.Revista Bistua.2016.14(1):112-124

+ Autor para el envió de correspondencia y la solicitud de las separatas: Luis Enrique Mendoza. Docente Universidad de Pamplona. Facultad de Ingenierías y Arquitectura, Programa de Ingeniería Mecatronica. Universidad de Pamplona. email: luismendoza@unipamplona.edu.co. 


\section{Introducciòn}

Desde tiempo atrás, ha existido un genuino interés por relacionar los aspectos del lenguaje con la mente, el cerebro y el pensamiento. Los estudios llevados a cabo por Broca, Wernicke y Lichtheim en la segunda mitad del siglo XIX fueron fundamentales para establecer conexiones entre el lenguaje y el cerebro a partir de dificultades en la codificación y decodificación del lenguaje manifestadas en diferentes pacientes ${ }^{1}$. El lenguaje es una capacidad humana específica que se manifiesta en forma de conducta observable consistente en signos verbales o de otro tipo, como los gestuales y tiene una realidad mental, fisiológica, psicológica y biológica ${ }^{2}$, parte de un cognitivo complejo que gira, por un lado, en torno al proceso de comprensión, definido como la capacidad de entender e interpretar el lenguaje hablado $y$ en el que intervienen factores cognitivos (atención, memoria, percepción) involucrados para llegar a la interpretación de unidades macro y microestructurales, que suministren producción, referida al proceso de programación y realización de la acción de hablar ${ }^{3}$. Las oscilaciones registradas en el EEG se producen por potenciales postsinapticos excitadores e inhibidores, desencadenados colectivamente por las columnas celulares corticales. La disposición estándar en rejilla de los electrodos de registro permite el muestreo global de la actividad eléctrica de forma que puede aplicarse a niños y a adultos ${ }^{4}$. Las lesiones que afectan al área de
Wernicke en adultos se asocian a la afasia receptiva ${ }^{3}$. En 1861, el patólogo francés Pierre Broca asignó la función motora del habla al giro frontal inferior del lado izquierdo, La principal área ocupa las porciones opercular y triangular del giro frontal inferior, correspondientes a las áreas 44 y 45 de Brodmann. Estas dos áreas son las que regulan la interpretación y gesticulación del lenguaje las cuales darán los impulsos neuronales, con previo acondicionamiento, filtrado y trasmisión la capacidad de efectuar técnicas robustas de procesamiento que brinden a un grupo de pacientes la capacidad de expresar o comunicarse con otros ${ }^{4}$.

\section{AFASIA}

La afasia es un trastorno de la función del lenguaje causado por una lesión cerebral. La fuente habitual es un accidente cerebrovascular producido por la oclusión vascular de la arteria cerebral media izquierda. Durante el habla se observa actividad en varias áreas de la corteza, sobre todo en el lóbulo frontal ${ }^{4,5,6}$. En la afasia de broca o motriz contiene distintos componentes, que provocan varias combinaciones. Los pacientes por definición presentan una afasia no fluente, ellos hablan pausadamente sin mucha entonación pero tienen dificultad para producir el habla espontanea, para denominar y repetir. Con este criterio se tiene diversos tipos de afasia motriz que dependen directamente del estado del paciente la edad algunos factores de interacción 5,7 . 
pueden detectarse voltajes oscilatorios

\subsection{Tratamiento Médico en afasias}

Las técnicas o procedimientos médicos para la corrección de afasias son obtenidos de pruebas y casos clínicos, de los pacientes que acuden dependiendo la sintomatología, pero todo comienza con evaluar el tipo de discapacidad en el lenguaje y de este modo entregar el diagnóstico, para aplicar una respectiva terapia con supervisión en los procesos, siempre y cuando se determinen metas factibles, para demuestra el proceso continuado 10. A partir del estudio médico, se encuentra Honner y Cols (1994), donde se relaciona las bases teóricas que sustentan los tratamientos, estas tres teorías son:

Tratamiento de estimulación por facilitación

Tratamiento neuropsicológico

Tratamiento de comunicación funcional

En los tratamientos convencionales de afasia, lo que se busca es estimular el lenguaje, mediante comprensión auditiva para disminuir el déficit, podemos llegar a tener terapias de acción visual y terapias de control voluntario ${ }^{10}$.

\section{SEÑALES ELECTRO- ENCEFALOGRAFÍCAS (SEEG)}

Desde su desarrollo inicial, el EEG sigue siendo la única herramienta para el estudio de la función cortical y un valioso complemento del historial clínico, la exploración física y la información obtenida en los estudios radiológicos. Cuando se colocan pequeños electrodos de disco metálico en la superficie del cuero cabelludo, de 20-100 $\mu \mathrm{V}$ que se conocen como electroencefalograma (EEG). Su origen es una consecuencia directa del efecto aditivo de grupos de neuronas corticales piramidales que se organizan en columnas radiales (dirigidas hacia el exterior). En este contexto, las columnas relevantes son las que están situadas debajo de la superficie de los giros corticales. Como los potenciales de membrana de estas columnas fluctúan, se desarrolla un dipolo eléctrico (áreas adyacentes de carga opuesta) $6,8,9$. Las oscilaciones registradas en el EEG se producen por potenciales postsinapticos excitadores e inhibidores, desencadenados colectivamente por las columnas celulares corticales. La disposición estándar en rejilla de los electrodos de registro permite el muestreo global de la actividad eléctrica de forma que puede aplicarse a niños y a adultos ${ }^{4}$, Las frecuencias y amplitudes de las señales electroencefalografías están en rangos bajos en frecuencia, hablamos de $0.5 \mathrm{~Hz}$ a $50 \mathrm{~Hz}$ y en voltaje desde $10 \mu \mathrm{V}$ a $100 \mu \mathrm{V}$

\section{ADQUISICIÓN, PROCESAMIENTO Y CARACTERIZACIÓN}

Para el desarrollo del trabajo se acude a escalas o procedimientos que logran que cada etapa sea idónea y entregue los resultados esperados. Se debe realizar un tamizaje en los pacientes, lo cual consiste en determinar unas cualidades o problemáticas comunes, que suministraran lecturas correctas o relacionadas a las señales provenientes de nuestros puntos de interés, por esta razón la población de prueba debe estar definida mediante edades y estados psicológicos que se hacen a partir de pruebas fisiológicas y psicológicas. Se parte del 
116

condicionamiento del paciente, mediante estímulos visuales y pronunciación de la letra para obtener como resultado un grupo de señales con semejanzas, esto se consigue con un paciente en disposición de la prueba con un estado de vigilia optimo, que brindan la capacidad de obtener señales con cambios donde deben ocurrir y no variaciones por diferentes aspectos en la toma de las señales, como ruidos mala postura que conllevan a cansancio o fatiga que se representa como errores 0 poca similitud entre las señales captadas.

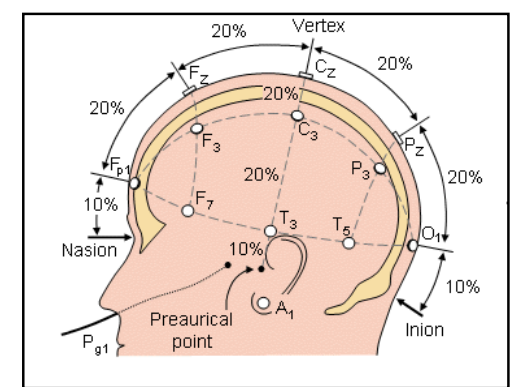

Fig. 1. Normativa 10/20 sistema internacional para ubicación de electrodos ${ }^{11}$.

Para la ubicación de los electrodos de tipo superficial se relacionan la normativa del sistema internacional 10 20, con las áreas de Brodman que encasillan el comportamiento eléctrico cerebral con nuestras señales de interés, 'para este caso particular la capacidad de obtener un carácter, una figura o una palabra que el paciente desee expresar. En la fig1. Se puede observar los puntos de interés para este caso las áreas de Brodman comprendidas para Wernicke, visión primaria y movimiento primario, para la normativa SI 10-20 se tiene, FT3,Fz,O5 respectivamente y un punto de referencia para este caso la parte inferior al lóbulo de la oreja que estaría designada con la letra $A 1$, este punto de referencia puede ser el mismo para la captura de más señales, pero para este caso particular solo se tomaran aquellas referentes al área de movimiento primario. En la fig.2 se puede detallar la forma del casco o diadema que tendrá el paciente en los puntos de control deseados y de este modo tomar las señales de maneja optima en cada instante de tiempo. Estas señales deben pasar por filtros en modo pasa-banda, con frecuencias de corte igual a: $0.5 \mathrm{~Hz}$ y $10 \mathrm{~Hz}$ que hacen parte de las frecuencias que se generan en la cabeza del ser humano 11.

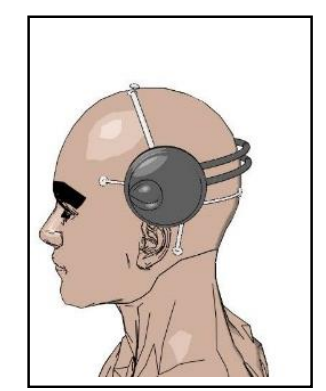

Fig. 2. Puntos de interés sobre la cabeza del paciente.

Estas señales tomadas del cerebro del paciente, antes de efectuar algún proceso de filtrado, se deben amplificar a rangos óptimos que suministren a los entornos computacionales la mayor cantidad de datos, evitando las perdidas y la inclusión de ruido, por esta razón se unan amplificadores de instrumentación biomédica. Los rangos de voltaje son relativamente bajos, señales en estado de vigilia están en el rango de $10 \mu \mathrm{V}$ a $100 \mu \mathrm{V}{ }^{11}$.

Para este caso la empresa Texas Instruments, ofrece componentes que trabajan este tipo de áreas y por cualidades se hace una elección del amplificador operación para 
instrumentación médica, INA 826, a continuación se muestra algunas de las cualidades 0 propiedades relevantes de este integrado ${ }^{12}$.

- Low Offset Voltage: $150 \mu \mathrm{V}$, $\max$

- Gain Drift: $1 \mathrm{ppm} /{ }^{\circ} \mathrm{C}(\mathrm{G}=1), 35$ $\mathrm{ppm} /{ }^{\circ} \mathrm{C}(\mathrm{G}>1)$

- Bandwidth: $1 \mathrm{MHz}(\mathrm{G}=1), 60$ $\mathrm{kHz}(\mathrm{G}=100)$

- Supply Current: $200 \mu \mathrm{A}$

- Supply Range: $2.7 \mathrm{~V}$ at $36 \mathrm{~V}$.

La arquitectura interna del amplificador de instrumentación, se muestra en la fig.3 donde se observa que el circuito integrado se compone de 4 amplificadores operaciones que proporcionan un aumento en la señal de manera instrumental minimizando perdidas y consumo energético ${ }^{12}$.

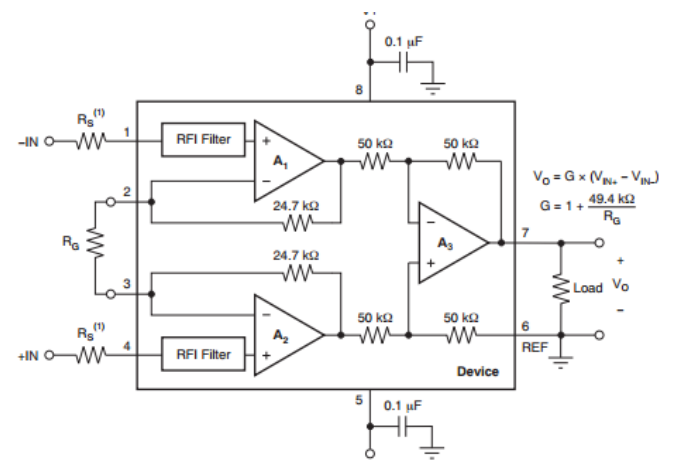

Fig. 3. Estructura interna del INA826. (Instruments)

De este modo la señal estará acorde a unos rangos óptimos, que podrán ser filtrados, en las frecuencias anteriormente descritas, donde se envían a un ordenador que realizara algoritmos en la búsqueda de los patrones característicos de lo que el paciente desea expresar. Para este caso cada señal obtenida pasara por un análisis wavelet, el cual, dependiendo los niveles de
117

descomposición se logre descartar las componentes de ruido y centrarse en las variaciones de cada impulso EEG.

Cuando se procesan las señales biomédicas, se tiene problemas ay que no son de tipo estacionario, por esta razón el uso de Fourier queda descartado, mientras que wavelet entrega la capacidad de tener una buena resolución temporal a altas frecuencias y a su vez buena resolución en frecuencias con registros largos. Donde la resolución varía según el plano tiempo-frecuencia, que le permite adaptarse a los cambios de cada señal ${ }^{13}$. La señal proveniente de los electrodos se denota por $x(t)$, se muestrea a fs Muestras/ segundo, creando una señal temporal discreta $x[n]$, de $N$ muestras, cuya máxima frecuencia puede ser fs./2. Dicha señal se pasa por un filtro paso-alto de media banda. $g[n]$, y por otro paso-bajo $h[n]$.según la regla de Nyquist, la mitad de las muestras se pueden eliminar después del filtrado, debido a que la frecuencia más alta que tendremos en este momento seria fs./4.asi se obtiene un nivel de descomposición, si denotamos a $\mathrm{Y}$ alto[k] e $\mathrm{Y}$ bajo[k] las salidas de los filtros paso-alto, paso bajo, después del sub-muestreo se expresa como:

$$
\begin{aligned}
& Y_{\text {alto }}=\sum_{n} x[n] \cdot g[2 k-n] \\
& Y_{\text {bajo }}=\sum_{n} x[n] \cdot h[2 k-n]
\end{aligned}
$$

Se usó el termino Wavelet para definir las funciones para muestrear una señal que se desea analizar en este caso EEG, y se propone la ecuación (3).

$$
S(\tau, a)=\int_{-\infty}^{\infty} s(t) \frac{1}{\sqrt{a}} \varphi *\left(\frac{t-\tau}{a}\right) \cdot d t
$$


Donde $\varphi *$ es el conjugado de la wavelet madre que será escalada y corrida punto a punto para determinar los niveles de comparación de la señal $\mathrm{s}(\mathrm{t})$. El valor de $\mathrm{a}=\frac{\mathrm{f}}{\mathrm{f}_{\mathrm{o}}}$, da la escala o dilatación de la wavelet, con un fo como frecuencia central y un $\tau$ el corrimiento 0 traslación en el tiempo.[15]

Existen gran variedad de Wavelet madre agrupadas en familias según su utilidad, Ingrid Daubechies quien es el mayor constructor de wavelets ha propuesto tres familias, la daubechies que es un conjunto de wavelets ortogonales apropiadas para aplicarse en análisis de señales discretas, la Coiflests llamada así por ser construidas a solicitud de R.coifman y la Symmlet que siendo similares a las daubechies tienden a ser casi simétricas, teniendo como base wavelet madre similares a la señal EEG de interés en este trabajo ${ }^{14}$.

\section{RESULTADOS}

Mediante el dispositivo electrónico para la extracción de señales biomédicas, PowerLab 26T fabricado por AD INSTRUMENTS se posicionan un par diferencial de electrodos sobre la zona relacionada al movimiento primario donde se extraen las señales con intervalos de tiempo comprendido entre 0 y 5 segundos, los pacientes poseen las mismas características de vigilia y tiempo de exposición, estos factores son relevantes debido a que cualquier tipo de distracción o fatiga puede arrojar errores en el proceso de adquisición. En la fig. 4 se observa un ejemplo de los pacientes con los electrodos en el área de interés, el dispositivo es configurado con
118

parámetros de filtrado, valor de amplitud y canal para que la señal tenga las características necesarias para ser trasmitida a un ordenador donde se llevarán a cabo técnicas digitales de procesamiento de señales. En este caso:

- Tiempo: 5 segundos

- Amplitud: $200 \mu \mathrm{V}$

- Filtro: $[0.5 \mathrm{~Hz}-20 \mathrm{~Hz}]$

- Frecuencia de Muestreo: $200 \mathrm{~Hz}$

- Samples:1280

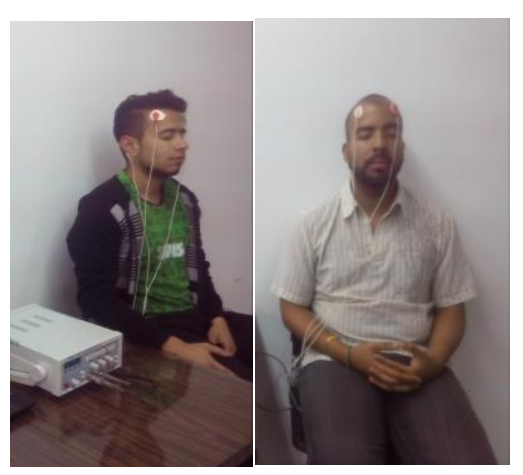

Fig. 4. Sujetos de prueba con electrodos en área de movimiento primario.

En esta fase el dispositivo PowerLab 26T otorga las capacidades de adquisición óptimas para estudiar las señales, pero a partir de resultados paulatinos el sistema será actualizado, implementando fases de amplificación, filtrado y adquisición, con la finalidad de minimizar espacio, peso y el tiempo de configuración del dispositivo de trabajo actual. La señal vista en la Fig.5, es capturada a partir de electrodos superficiales ubicados sobre el paciente o sujeto de prueba sobre el área de Brodman que comprende el movimiento primario, para este caso el área frontal de cerebro, debido a que los procesos de gesticulación, con un condicionamiento previo, respecto a 
luminosidad, posición y estado de relajación del paciente.

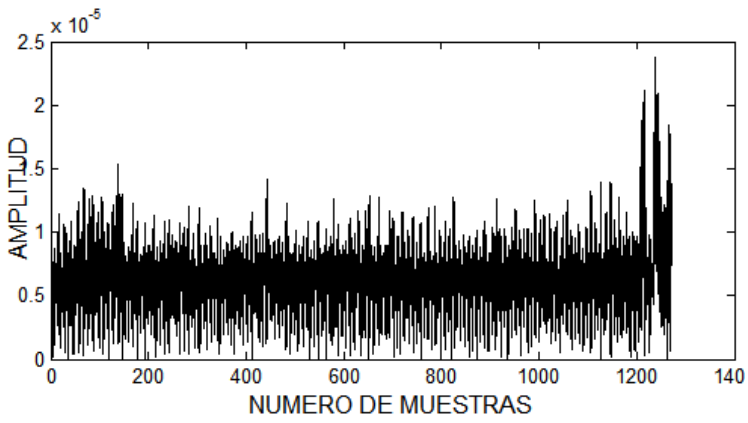

Fig. 5. Señal de reposo adquirida de área de movimiento primario.

La primera actividad que el paciente debe realizar es la fonética 0 pronunciación de la letra " $A$ ", con un tiempo de 5 segundos, donde el área de movimiento está relacionada con cambios gesticulares 0 fonéticos, debido a la vibración de las cuerdas vocales y los cambios faciales que se presenten al pronunciar la letra.En la Fig.6 se observa dos señales provocadas con la pronunciación de la letra "A", donde se puede ver un cambio respecto a la señal de reposo, y una zona de interés característica en todas las señales tomadas, para este caso existe una amplitud notable en la señal color rojo y en la señal de color negro, con una cantidad de muestras, óptimas para el tiempo de adquisición y una frecuencia de muestreo de $200 \mathrm{~Hz}$ con una amplitud estipulada fija para las practicas tanto en fonética como en escritura de la letra "A".

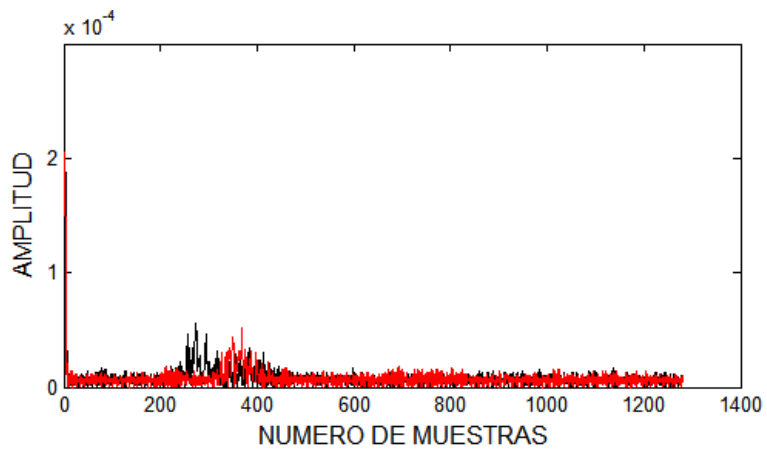

119

Fig. 6. Señales registradas al pronunciar la letra "A".

En la fig.7, se observa la aplicación de una trasformada Wavelet de coeficientes de aproximación con una madre Daubechies 5, "db5", la señal obtenida al pronunciar la letra "A", se toma dicha Wavelet madre por la similitud de SEEG, aplicando tres niveles de descomposición donde existe una reducción en la cantidad muestras, donde la señal original contiene 1280 , en un primer nivel se tendrán 640 , segundo 320 y en el tercer nivel se tendrán 160 muestras, donde se pueden apreciar cambios debido a que wavelet es una trasformada en base a un banco de filtros.

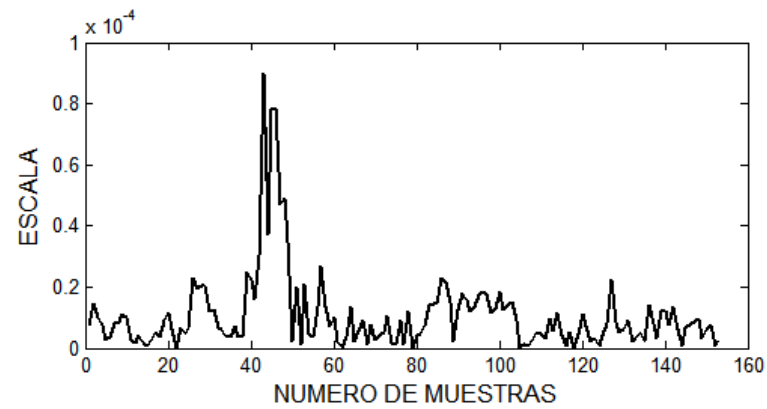

Fig. 7. Aplicación de Wavelet coeficiente aproximación sobre señal de letra "A" pronunciada.

Para el segundo caso se toman las mismas consideraciones de posición del paciente pero se hace una variación en el impulso cerebral esperado, para este caso el paciente debe escribir la letra "A", en los cinco segundos estipulados anteriormente, dos veces para establecer zonas de interés que servirán como guía para la extracción de las características, estas señales puede ser apreciada en la fig.8, con un total de 1280 muestras, previamente el paciente es condicionado para que dibuje siempre 
la misma letra "A" con un tamaño aproximado en cada una de las veces. La señal de color rojo es el registro número 1 y la señal negra es el registro 10.

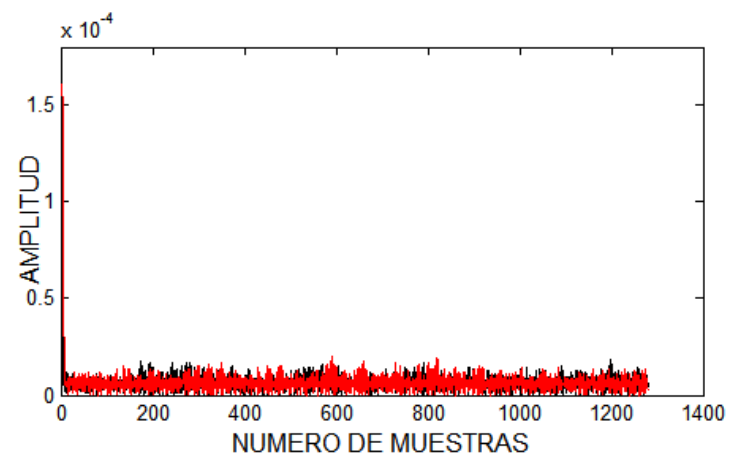

Fig. 8. Señal de producida al escribir la letra "A".

Mediante la transformada Wavelet, con coeficientes de aproximación se logra percibir aquellas variaciones en la escritura de la letra "A", con respecto a la señal de reposo, se efectúan tres niveles de descomposición al igual que en caso anterior, se puede observar en la Fig.8 donde las componentes de baja frecuencia son atenuadas por cada función del banco de filtros, el número de muestras disminuye en la misma proporción, para este nivel se tiene, 160 muestras, pero sin perder la forma de la señal original, que dará paso a la búsqueda de los patrones característicos.

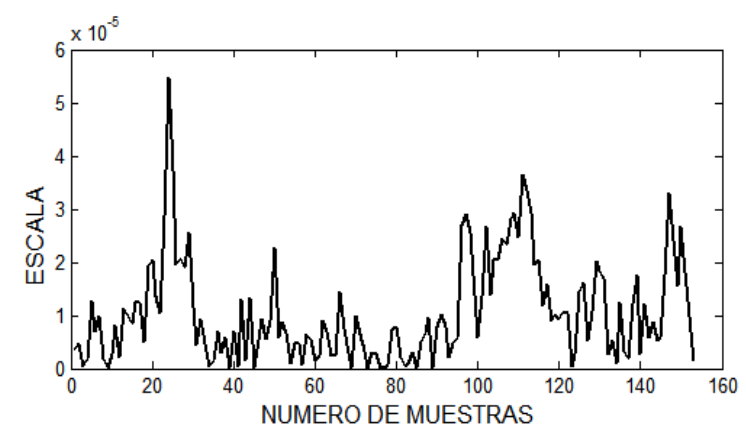

Fig. 9. Aplicación de Wavelet coeficiente aproximación sobre señal de letra "A" escrita
Las señales registradas en las dos actividades, pronunciar y escribir la letra presentar variaciones notables, aunque para obtener una o más zonas de interés se tiene como referencia una señal de reposo, la cual está relacionada directamente con una actividad cerebral normal sin perturbaciones de sueño, ruidos externos o cualquier otro factor que pueda afectarla, también se debe aclarar que la forma de onda vista en las Fig.6, Fig. 8 fue repetitivo en la mayoría de las señales tomadas lo que afirma que existen impulsos eléctricos provenientes del área de Brodman vinculada al movimiento primario cuando se gesticula y cuando se mueve el brazo para escribir la letra, para este caso puntual la letra "A". En base a los datos de cada una de las señales tomadas y estableciendo puntos de interés, respecto a la señal de reposo en la fig.10 se muestra la zona de interés encerrada en un rectángulo, para la señal formada al pronunciar la letra "A" donde los tres niveles de descomposición de la señal mediante la transformada Wavelet otorgan mejor capacidad de percepción eliminando componentes de ruido relacionadas con las altas frecuencias, recordando que las SEEG son inferiores a $10 \mathrm{~Hz}$.

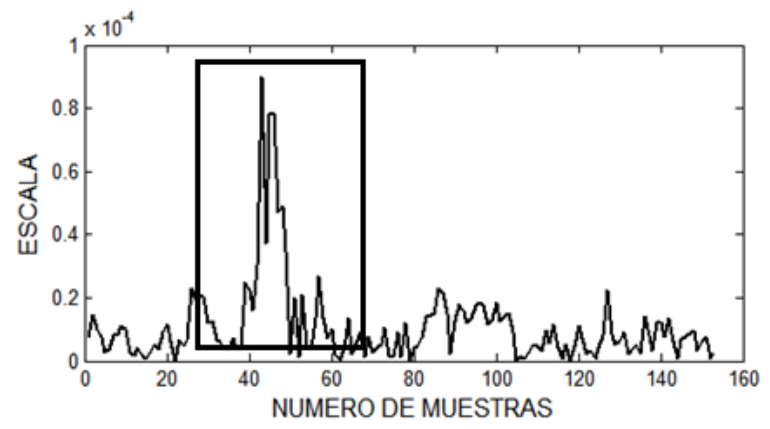

Fig.10 selección de zona de interés de señal pronunciando la letra " $A$ " 
Realizando el procedimiento anterior con otra señal generada al pronunciar la letra "A", la zona de interés se ve reflejada de nuevo en la fig.11, con un factor de escala y comprendida entre el rango de muestras de la fig. 10.

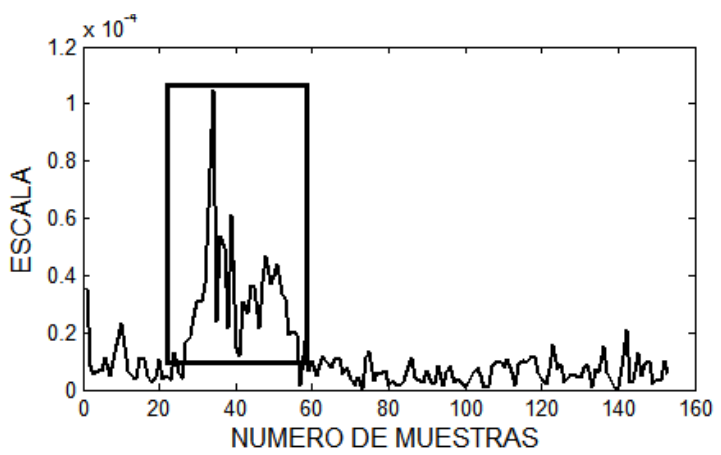

Fig.11 selección de zona de interés de señal pronunciando la letra "A"

De igual modo se hace un análisis de la forma de la señal para determinar áreas de interés al ejecutar la acción de la escritura de la letra " $A$ ", apoyados en la eliminación de frecuencias que realiza la transformada Wavelet, en coeficientes de aproximación con tres niveles de descomposición, en la Fig.11 se indican las dos zonas de interés que se forman al realizar el movimiento de la extremidad superior derecha para escribir la letra "A", donde cada uno de los puntos de interés está encerrado en un rectángulo.

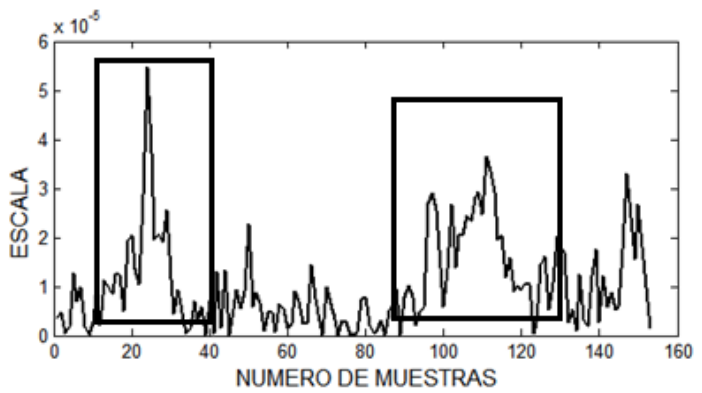

Fig.12 Selección de zona de interés de señal escribiendo la letra " $A$ ".

En la Fig.13 se puede apreciar otra señal producida por la escritura de la letra "A", donde las zonas de interés
121

vuelven a tener relación con fig.12 estipulando un espacio para la extracción de los patrones asociados a la actividad de escritura de la letra "A".

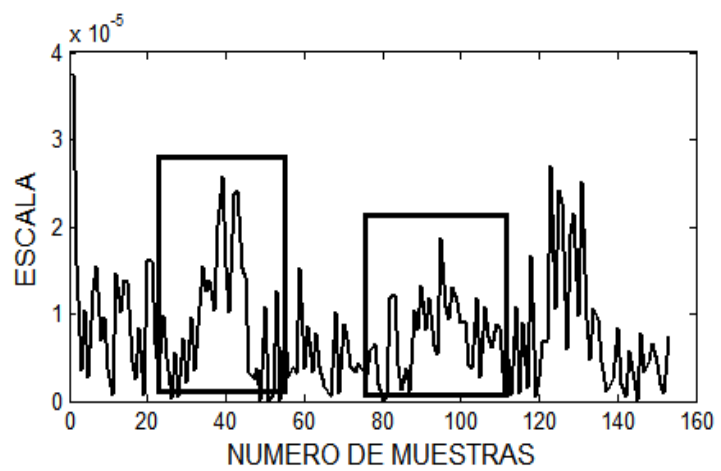

Fig.13 Selección de zona de interés de señal escribiendo la letra "A".

Dentro de las zonas de interés relacionadas en las Fig10, Fig12 la actividad cerebral varia en amplitud o en la fuerza que tenga la señal, se debe recordar que la configuración de amplitud del dispositivo de adquisición PowerLab 26T, fue ajustada en $200 \mu \mathrm{V}$, con un filtrado entre $[0.5 \mathrm{~Hz}-20 \mathrm{~Hz}$, este tipo de variaciones en cada una de las señales demuestra que existe diferencia entre los procesos cognitivos y los procesos de movimiento de un sujeto de pruebas o paciente, para este caso dos personas con rangos de edad similares, en condiciones de vigilia entre otros.

Estos cambios en la actividad cerebral, en las dos actividades pronunciación y escritura de la letra "A", pueden dar espacio a tratamientos de afasia motriz los cuales en etapas preliminares buscan que el paciente relacione los pensamientos con la actividad del lenguaje, existen variaciones entre cada uno de los impulsos que deben ser filtradas a partir de bancos de filtros que otorga la transformada Wavelet y que se convierten en herramientas útiles, en los procesos de búsqueda de 
patrones con cada uno de los impulsos obtenidos de cada uno de los pacientes. Inicialmente las señales son tomadas con electrodos superficiales $\mathrm{Ag} / \mathrm{ClAg}$ con sustancia adherente, debido a que no se están usando todas las áreas de Brodman descritas inicialmente, pero en base al área de movimiento primario se relacionan los procesos cognitivos a los proceso de tipo motriz que permiten que el paciente gesticule, mueva los músculos faciales y mueva sus extremidades superiores al momento de escribir. Para efectos futuros de la toma de las señales EEG se hace necesario el uso de un casco o diadema que tenga las medidas de las áreas de Brodman de interés para la extracción de las señales, el modelado se realiza a partir de herramientas de software como rhinoceros, que brindan la capacidad de medidas reales y visualización 3D del dispositivo 0 mecanismo deseado.

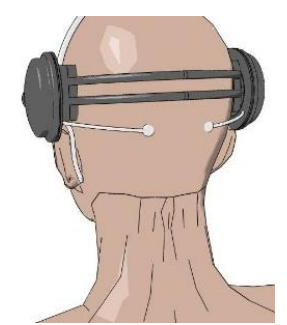

Fig. 14. Parte posterior diadema de adquisición

En la fig.14 se muestra la cabeza del paciente en modelado 3D, relaciona el área de visión primaria con dos electrodos dispuestos de forma diferencial en color blanco, y el electrodo de referencia ubicado debajo de la posición del canal auditivo, u oreja izquierda., el cuerpo de la diadema es aquel de color negro, el cual da la capacidad de amarre a los electrodos con sus respectivos conductos, esta estructura debe ser lo
122

elástica, cualidad que se verá reflejada en la facilidad de acople con los pacientes, y a su vez la correcta ubicación de los electrodos en cada espacio de adquisición. Se debe efectuar un proceso de render sobre el modelo hecho en rhinoceros para tener una semejanza al real, en la Fig. 15 se aprecia la diadema en las diferentes vistas con los electrodos superficiales de color dorado y el electrodo de referencia de color blanco, y unos conductos designados por el color azul.

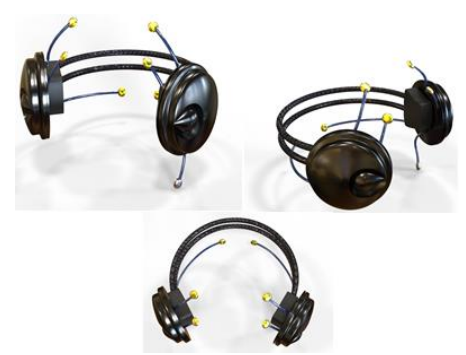

Fig. 15. Vistas del modelo 3D

Hasta el momento se tienen señales de diez sujetos con capacidades normales de dialogo, pero debido a los puntos de interés que se mencionan en el trabajo, los resultados deben ser similares a los de una persona que muestre síntomas de afasia, las señales de registro que se han tomado son referentes a, evocación de letras, gesticulación o vocalización, y movimiento de extremidades, las anteriores señales con puntos de referencia en el área craneal frontal, relacionada con el movimiento primario descrito por Brodman.

\section{CONCLUSIONES}

Este trabajo logró registrar SEEG, en las cuales se caracteriza la pronunciación y la escritura de la letra "A" en diez pacientes o sujetos de prueba. Se realizó este procedimiento 
con el fin de demostrar que las SEEG, mantienen patrones o características, donde se pueda establecer un área de interés para la búsqueda de información relacionada con la fonética y la escritura de la letra "A", y un futuro realizar sistemas inteligentes con los datos obtenidos apoyados en la utilidad que brinda la transformada Wavelet en el filtrado ,para una posterior extracción de patrones y la compresión de información, y de esta manera crear un mapa de patrones que permitan reconocer palabras y gestos en personas con problemas de afasia motriz. Se demostró que los patrones relacionados con escritura y fonética de la letra "A", fue visualizado en el $100 \%$ de los datos registrados.

Finalmente es importante resaltar que se deben seleccionar áreas del cerebro relacionadas a los procesos cognitivos motores, apoyados en Brodman que permitan tener más registros de SEEG, y de esta manera incrementar los niveles de exactitud y precisión de un sistema que permita una alternativa de comunicación para aquellas personas que no tienen la posibilidad de comunicarse de manera fonética, o que han perdido la capacidad de hablar por problemas en el área de Broca.
123

\section{Referencias bibliográficas}

[1] Fernández, M. "El carácter de la ciencia lingüística”, Verba, 11, págs. 129-156. 1984.

[2] Alonso-Cortés, A. Lingüística. Madrid, Cátedra. 2002.

[3] Cummings, L. Clinical linguistics. Edimburgo, Edimburg University Press. 2008.

[4] FitzGerald, M. J. Neuroanatomía clínica y neurociencia. España: Elsevier. 2012.

[5] H. G. Sanchez J, «Afasia, Un trastorno del lenguaje de fisiopatologia compleja y de origen multifactorial,» Revista de Especialidades MédicoQuirurgicas, vol. 8, o 1, p. 9, 2003.

[6] Waxman, s. g. Neuroanatomia clinica. Mac Graw hill. 2011.

[7] Thakor, n. IEEE lifesciences. Recuperado el 22 de 04 de 2015, de building brain machine interfaces speech and comunication: http://lifesciences.ieee.org /publications/newsletteray-2012/103building-brain-machine-interfacesspeech-language-and-communication. 2013.

[8] Utianski, R. L. Cortical characteriza-tion of the perception of intelligible. Brain \& Language, 48-54. 2014.

[9] Hausfeld, L. Pattern analysis of EEG responses to speech and voice: 
Influence of feature grouping.

Neurolmage, 3641-3651. 2012.

[10] S. N. Saa, «Rehabilitacion de la afasia,» Cuadenos de Neurologia, vol. 25, p. 6,2001

[11] Malmivuo, J. (s.f.). Bio electromagnetism, de http://www.bem.fi/. 2015.

[12] Instruments, T.(s.f.). TeXas Instruments. Recuperado el 29 de 03 de 2015, de http://www.ti.com/lit/ds/symlink/ina826. pdf

[13] A. Figliola and E. Serrano, "Analysis of Phisiological Time Series Using Wavelet Transforms", IEEE Eng. Med. Biol. Mag., 16(3),pp. 74-79, MayJun. 1997.

[14] D. A. G. S. Jesus Poza,. [En línea].

Available:

http://www.researchgate.net/publicatio n/267971643_Anlisis_de_Electroencef alogramas_con_la_Transformada_Wa velet_Discreta._Aplicacin_a_la_Enfer medad_de_Alzheimer 2015.

[15] R.C.Acevedo «Extracción de características interfaces cerebro computadoras mediante trasformada wavelet discreta» Memorias del XVII congreso Argentino de Bioingeniería No 167 p 58-62, 2009. 